\title{
Increased distance between mitral valve coaptation point and mitral annular plane: significance and correlations in patients with heart failure
}

\author{
S E Karagiannis, G T Karatasakis, N Koutsogiannis, G D Athanasopoulos, D V Cokkinos
}

Heart 2003;89:1174-1178

See end of article for authors' affiliations

Correspondence to: Dr George Karatasakis, Onassis Cardiac Surgery Centre, 356 Syngrou Ave, Athens 17674, Greece; georgekar2001@yahoo.com

Accepted 22 April 2003

\begin{abstract}
Objective: To measure the distance between the mitral leaflet coaptation point and the mitral annulus (CPMA) and assess the relation of this index to structural and functional characteristics of the failing left ventricle.
\end{abstract}

Design: Echocardiographic indices and CPMA were measured at baseline and again during dobutamine infusion and leg lifting. Left ventricular diastolic and systolic dimensions, left ventricular ejection fraction (LVEF) by Simpson's rule, mitral annulus dimension, and E point septal separation were correlated with CPMA.

Setting: Tertiary referral centre.

Patients: The total study population of 129 patients included 94 with LVEF < 35\% and 35 with LVEF $35 \%-45 \% ; 76$ had coronary artery disease and 53 had dilated cardiomyopathy.

Interventions: A dobutamine infusion was given in 18 patients and preload increase by leg lifting in 28.

Main outcome measures: Correlations between CPMA and contractility indices at baseline and during interventions.

Results: CPMA was correlated with left ventricular diastolic dimension ( $r=0.52)$, left ventricular systolic dimension $(r=0.53), \operatorname{LVEF}(r=-0.44)$, fractional shortening $(r=-0.42)$, E point septal separation $(r=0.48)$, and mitral annulus dimension $(r=0.44)$ (all $p<0.001)$. Dobutamine decreased CPMA from (mean (SD)) 12.04 (3.64) $\mathrm{mm}$ to 8.92 (2.56) $\mathrm{mm}$ and increased LVEF from 27 (6.2)\% at baseline to $33.4(6.9) \%$ at $10 \mathrm{\mu g} / \mathrm{kg} / \mathrm{min}$ (both $p<0.001)$. These changes were strongly related $(r=0.68$, $p<0.007)$. After leg lifting, CPMA decreased from 13 (4) $\mathrm{mm}$ at baseline to 10 (3) $\mathrm{mm}(\mathrm{p}<0.001)$, and LVEF increased from $32(11) \%$ at baseline to $39(11) \%(p<0.001)$. Fractional shortening and left ventricular diastolic dimension also increased $(p<0.001)$ and mitral annulus dimension and $E$ point septal separation decreased ( $p<0.002)$, but left ventricular systolic dimension did not change.

Conclusions: The mechanism displacing the mitral coaptation point towards the left ventricular apex is multifactorial. The correlations between CPMA difference (before versus after interventions) and ejection fraction difference (before versus after interventions) shows that this index depends mainly on left ventricular function.
U nder normal conditions, the coaptation point of the mitral valve leaflets in systole practically reaches the level of the mitral annulus. This point is displaced apically in abnormal conditions, such as morphological abnormalities of the leaflets or dilatation of the left ventricle. As a result the distance between the coaptation point of the leaflets and the level of the mitral annulus is increased.

This finding is described as incomplete mitral leaflet closure and may be the result of failure of one or both leaflets to reach the level of the atrioventricular ring at the point of its peak systolic movement. It has been attributed to papillary muscle dysfunction, raised left ventricular filling pressure, and left ventricular dysfunction..$^{1-5}$ The first to describe this occurrence were Ogawa and colleagues ${ }^{1}$ and Godley and associates ${ }^{2}$ in patients with papillary muscle dysfunction. Kinney and Frangi $i^{3}$ placed greater emphasis on left ventricular function and on raised left ventricular filling pressures. Kaul and colleagues, in both clinical ${ }^{4}$ and experimental ${ }^{5}$ studies, attributed the finding to poor left ventricular systolic function rather than to papillary muscle dysfunction.

Two theories have been proposed to explain the mechanism of incomplete mitral closure in patients without intrinsic valvar abnormalities. According to the "regional" theory, in ischaemic heart disease there are geometrical changes in the mitral leaflet attachment region or at the base of the papillary muscles producing traction of dyskinetic areas that prevents the mitral leaflets from coapting fully. ${ }^{2-6}$

The alternative is the "global" theory. ${ }^{7}$ Incomplete mitral leaflet closure often coincides with restricted diastolic leaflet excursion in patients with left ventricular dysfunction. This restricted diastolic leaflet excursion reaches the tethering line connecting the mitral annulus and the papillary muscle and reflects limitation of anterior motion relative to the posteriorly placed papillary muscles, without a decrease in total orifice area. ${ }^{89}$ This increased tethering by the displaced mitral leaflet attachments can restrict both diastolic opening and systolic closure. According to the global theory, dilatation and a slow rise in systolic pressure are the causes of this phenomenon.

We prospectively measured the distance between the coaptation point and the mitral annulus (CPMA), which represents a quantitative estimate of incomplete mitral closure, to assess the anatomical or functional characteristics of the failing left ventricle to which this index is related.

Abbreviations: CPMA, distance betwen the coaptation point and the mitral annulus; EDD, end diastolic diameter; EDS, end systolic diameter; LVEF, left ventricular ejection fraction; NYHA, New York Heart Association 


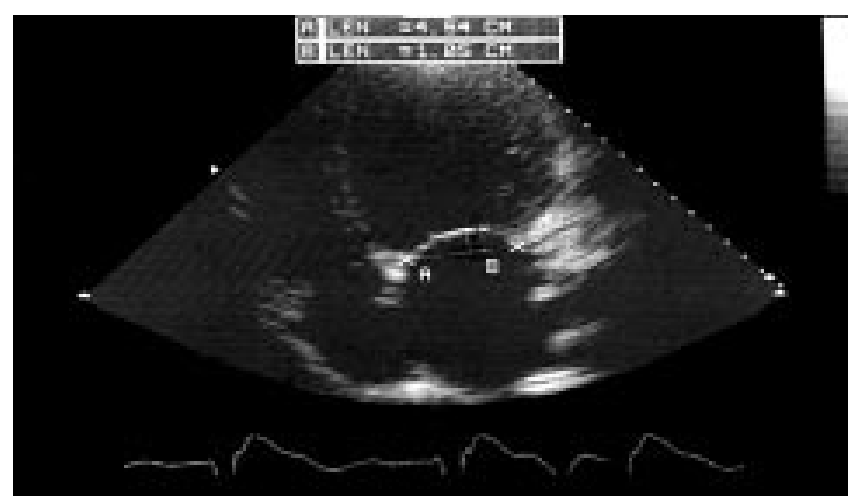

Figure 1 Two dimensional four chamber view, showing the mitral annular plane and its distance from the mitral leaflet coaptation point (CPMA).

\section{METHODS \\ Patients}

The study population consisted of 129 patients ( 117 men, 12 women), mean (SD) age 56 (25) years (range $21-82$ years) who were under evaluation and treatment for clinical heart failure. Only patients in New York Heart Association (NYHA) functional class III or IV were included.

In 76 patients $(59 \%)$ the cause of the heart failure was coronary artery disease, defined as more than $70 \%$ narrowing of at least one major coronary artery and diagnosed by coronary arteriography. In 53 patients $(41 \%)$ a diagnosis of dilated cardiomyopathy was established by excluding significant coronary or valvar heart disease.

The population was subdivided into two groups: group A comprised 94 patients who were known to have a left ventricular ejection fraction (LVEF) of $<35 \%$ on previous echocardiographic evaluation; group B comprised 35 patients with an LVEF of 35-45\%.

Patients with significant primary valvar heart disease were excluded. All the patients were under treatment with diuretics and angiotensin converting enzyme inhibitors, and 45 were also being treated with digoxin.

\section{Procedures}

All patients underwent a baseline prospective echocardiographic examination using an HP 2500 system (HewlettPackard Inc, Andover, Massachusetts, USA) equipped with a 2.5 MHz phased array probe. In two subgroups of the group A population, specific further manoeuvres were undertaken: dynamic echocardiography using a low dose dobutamine protocol in 18 patients, and preload increase by leg lifting in 28 . No patients in group B underwent this further study.

\section{Baseline study}

In all patients (groups A and B), left ventricular end diastolic (EDD) and end systolic (EDS) diameters and the E point septal separation were measured according to the recommendations of the American Society of Echocardiography. ${ }^{10}$ Fractional shortening was determined and the left ventricular ejection fraction was estimated by Simpson's rule. The mitral annulus diameter was measured from the apical four chamber view. From this view we also calculated the distance between the mitral leaflet coaptation point and the mitral annular plane (CPMA), during end systole (fig l).

The $\mathrm{E}$ and $\mathrm{A}$ waves of the transmitral flow velocity pattern, as well as the deceleration time of the $\mathrm{E}$ wave and the E/A ratio, were measured as the average over five consecutive beats. The existence and degree of mitral regurgitation were assessed semiquantitively. ${ }^{11}{ }^{12}$

\section{Dobutamine study}

From the group A study population, 18 patients, four with dilated cardiomyopathy and 14 with coronary artery disease, underwent a low dose dobutamine echocardiographic protocol with two intravenous dosages of dobutamine. Each stage lasted five minutes. At the end of each stage all baseline measurements were repeated. The dose of dobutamine was $5 \mu \mathrm{g} / \mathrm{kg} / \mathrm{min}$ in the first stage and $10 \mu \mathrm{g} / \mathrm{kg} / \mathrm{min}$ in the second.

\section{Preload increase study}

In 28 group A subjects, the preload was increased by lifting the legs at a $45^{\circ}$ angle and the measurements described above were repeated.

\section{Statistics}

All values are expressed as mean (SD). A probability value of $\mathrm{p}<0.05$ was considered significant. An independent samples $t$ test was used to compare groups of patients with dilated cardiomyopathy and coronary artery disease and to evaluate differences between patients with ejection fractions of $<35 \%$ or $\geqslant 35 \%$. A paired $t$ test was used to evaluate the effect of dobutamine administration and preload increase on the baseline characteristics. Parametric and non-parametric tests (Pearson and Spearman correlations) were applied to correlate CPMA changes produced by dobutamine administration and preload increase with functional and geometrical variables.

\section{RESULTS}

\section{Baseline measurements}

The demographic data and echocardiographic variables of the patients are shown in table 1. Patients with dilated cardiomyopathy had more significant left ventricular dilatation than patients with coronary artery disease. However, they did not

Table 1 Clinical and echocardiographic characteristics of patients with dilated cardiomyopathy and coronary artery disease, and of the patients forming group A (ejection fraction $<35 \%$ ) and group B (ejection fraction 35-45\%)

\begin{tabular}{|c|c|c|c|c|c|c|}
\hline Variable & $\begin{array}{l}\text { DCM } \\
(n=53)\end{array}$ & $\begin{array}{l}\text { CAD } \\
(n=76)\end{array}$ & $\begin{array}{l}\text { p Value } \\
\text { (DCM } \vee \text { CAD) }\end{array}$ & $\begin{array}{l}\text { Group A } \\
(n=94)\end{array}$ & $\begin{array}{l}\text { Group B } \\
(n=35)\end{array}$ & $\begin{array}{l}\text { p Value } \\
\text { (group } A \vee B \text { ) }\end{array}$ \\
\hline Men & 46 & 72 & & 82 & 35 & \\
\hline Women & 7 & 5 & & 12 & 0 & \\
\hline Age (years) & $48(13)$ & $62(11)$ & $<0.001$ & $57(14)$ & $58(13)$ & 0.66 \\
\hline Diastolic diameter $(\mathrm{mm})$ & 75 (10.7) & $68.6(9.2)$ & 0.001 & $73.6(10.2)$ & $65.1(8.0)$ & $<0.001$ \\
\hline Systolic diameter $(\mathrm{mm})$ & $62.4(12.6)$ & $56.8(9.8)$ & 0.009 & 62.3 (1 1.0$)$ & $50.6(7.0)$ & $<0.001$ \\
\hline Fractional shortening (\%) & $16.0(5.3)$ & $17.4(4.7)$ & 0.125 & $15.2(4.3)$ & $21.2(3.9)$ & $<0.001$ \\
\hline Ejection fraction (\%) & $28.2(7.9)$ & $30.1(6.9)$ & 0.153 & $<35$ & $35-45$ & * \\
\hline EPSS (mm) & $29.1(9.6)$ & $27.6(7.2)$ & 0.329 & $30.0(8.6)$ & $23.6(4.8)$ & $<0.001$ \\
\hline Mitral annular diameter $(\mathrm{mm})$ & $41.6(6.3)$ & $40.4(4.5)$ & 0.238 & $41.2(5.4)$ & $40(5.1)$ & 0.277 \\
\hline Coaptation point - mitral annulus diameter $(\mathrm{mm})$ & $12.1(3.1)$ & $11.2(2.8)$ & 0.099 & $12.1(3.0)$ & $10.3(2.5)$ & 0.001 \\
\hline
\end{tabular}

Values are mean (SD).

*Pre-specified difference.

CAD, coronary artery disease; DCM, dilated cardiomyopathy; EPSS, E point septal separation. 




differ in the systolic impairment or mitral annular dilatation. The CPMA was similar in the two aetiological groups.

Overall, there was a linear correlation between CPMA and measurements of left ventricular size (table 2)-thus the CPMA was significantly related to both EDD and EDS of the left ventricle. There was an inverse correlation between CPMA and indices of systolic performance of the left ventricle such as fractional shortening and ejection fraction, while E point septal separation and CPMA showed a positive correlation. Furthermore, CPMA was also related to the mitral annulus dimension. These correlations between variables of interest and CPMA were more significant in the patients with dilated cardiomyopathy than in those with coronary artery disease.

Conversely, there was no correlation between CPMA and echocardiographic indices of diastolic function - that is, with the $\mathrm{E}$ and A waves of the transmitral flow velocity pattern-in either group of patients. Additionally, the E/A ratio and E wave deceleration time were not related to CPMA.

The correlations found in group A patients were similar to the mean values for the whole study population. In group B patients, the CPMA was significantly smaller than in group A $(\mathrm{p}=0.001)$. The ejection fraction and fractional shortening were also greater, as predetermined by their selection, and these patients had significant differences in the ventricular systolic and diastolic dimensions and E point septal separation (table 1). Group B patients showed overall correlations between CPMA and ejection fraction $(r=-0.315, \mathrm{p}<0.035)$, E point septal separation $(r=0.511, \mathrm{p}<0.002)$, and mitral annulus dilatation $(r=0.371, \mathrm{p}<0.03)$, as in group A patients, but there was no significant correlation with fractional shortening $(r=-0.108, \mathrm{p}=0.539)$ or $\mathrm{EDD}$ $(r=0.179, \mathrm{p}=0.303)$ and EDS $(r=0.209, \mathrm{p}=0.229)$.

In relation to the degree of mitral regurgitation, in 28 $(21.7 \%)$ of our patients, mitral regurgitation of mild or moderate degree was found. These two subgroups (with and without mitral regurgitation) did not differ with regard to cavity dimension, E point septal separation, mitral annulus diameter, or CPMA, but the left ventricular ejection fraction and fractional shortening were lower in the mitral regurgitation group $(\mathrm{p}<0.001)$.

\section{Dobutamine administration}

In the 18 patients from group A who underwent the low dose dobutamine protocol, a significant dose dependent decrease in CPMA was noted, accompanied by an increase in fractional shortening and ejection fraction (table 3). The effects of dobutamine administration on ejection fraction and CPMA were strongly related. Specifically, the correlation between the difference in ejection fraction and the difference in CPMA before and after dobutamine administration was $r=0.68$ $(\mathrm{p}<0.014)$ (fig 2). Neither parametric nor non-parametric tests revealed any correlation between CPMA differences before and after dobutamine and changes in mitral annulus dimension, EDD, or ESD before and after dobutamine. The correlation coefficient values between CPMA difference and EDD and ESD differences using Pearson's parametric correlations were $r=-0.244(p=0.363)$ and $r=0.222(p=0.410)$, respectively. Using Spearman's non-parametric correlations the equivalent coefficients were $r=-0.217(\mathrm{p}=0.419)$ and $r=0.259(\mathrm{p}=0.332)$.

\section{Preload increase}

Preload increase in 28 patients from group A caused by leg lifting produced a significant decrease in CPMA and an increase in both ejection fraction and fractional shortening. EDD also increased, while E point septal separation and mitral annulus dimension decreased (table 4). These changes were similar to those produced by dobutamine administration.

However, in contrast to dobutamine, the difference in CPMA before and after leg raising was not correlated with differences in left ventricular dimensions and function. The correlations between the difference in CPMA before and after leg

Table 3 Functional cardiac variables at baseline and post-dobutamine

\begin{tabular}{llll}
\hline & & \multicolumn{2}{l}{ Dobutamine administration } \\
\cline { 3 - 4 } Variable & Baseline & $5 \mu \mathrm{g} / \mathrm{kg} / \mathrm{min}(\mathrm{n}=14)$ & $10 \mu \mathrm{g} / \mathrm{kg} / \mathrm{min}(\mathrm{n}=18)$ \\
\hline Diastolic diameter $(\mathrm{mm})$ & $70.18(11.20)$ & $66.10(11.57)^{*}$ & $68.00(11.34)^{*}$ \\
Systolic diameter $(\mathrm{mm})$ & $59.23(11.71)$ & $54.30(11.84)^{*}$ & $54.75(11.36)^{*}$ \\
Fractional shortening (\%) & $16.00(5.30)$ & $19.20(6.50)^{*}$ & $20.00(5.61)^{*}$ \\
Ejection fraction (\%) & $27.00(6.18)$ & $29.9(7.67)^{*}$ & $33.4(6.86)^{*}$ \\
EPSS (mm) & $29.13(6.60)$ & $26.35(7.01)^{*}$ & $24.81(7.38)^{*}$ \\
Mitral annulus diameter (mm) & $40.28(6.51)$ & $36.74(5.36) \dagger$ & $38.13(4.05) \dagger$ \\
CPMA (mm) & $12.04(3.64)$ & $9.08(2.57)^{*}$ & $8.92(2.56)^{*}$ \\
\hline Values are mean (SD). & & & \\
*p<0.001; †p<0.01 v baseline. & & & \\
CPMA, distance between the coaptation point and the mitral annulus; EPSS, E point septal separation.
\end{tabular}






Figure 2 Scatterplot of the difference in the mitral leaflet coaptation point (CPMADIF) before and after dobutamine administration $(10 \mu \mathrm{g} / \mathrm{kg} / \mathrm{min})$ against the difference in the ejection fraction (EFDIFF) before and after dobutamine $(r=0.68, p<0.007)$.

\begin{tabular}{llll} 
Table 4 & Values after leg lifting & & \\
\hline Variable & Baseline & Leg lifting & p Value \\
\hline Diastolic diameter $(\mathrm{mm})$ & $69(12)$ & $72(12)$ & 0.001 \\
Systolic diameter $(\mathrm{mm})$ & $58(13)$ & $58(13)$ & 0.51 \\
FS $(\%)$ & $17(6)$ & $21(7)$ & 0.001 \\
EF (\%) & $32(11)$ & $39(11)$ & 0.001 \\
EPSS (mm) & $30(8)$ & $25(8)$ & 0.002 \\
MAD (mm) & $42(4)$ & $39(4)$ & 0.002 \\
CPMA (mm) & $13(4)$ & $10(3)$ & 0.001 \\
\hline
\end{tabular}

CPMA, distance between the coaptation point and the mitral annulus; $E F$, ejection fraction; EPSS, E point septal separation; FS, fractional shortening; MAD, mitral annulus diameter.

raising and differences in ejection fraction, EDD, and EDS before and after leg raising were $r=0.152(\mathrm{p}=0.354)$, $r=-0.064(\mathrm{p}=0.731)$, and $r=-0.191(\mathrm{p}=0.303)$, respectively, with Pearson's parametric test; with Spearman's non-parametric test the equivalent values were $r=0.146$ $(\mathrm{p}=0.457), \quad r=-0.122 \quad(\mathrm{p}=0.537), \quad$ and $\quad r=-0.152$ $(\mathrm{p}=0.441)$.

\section{DISCUSSION}

The occurrence of incomplete mitral leaflet closure was initially thought to be caused by dyskinesia of the left ventricular myocardium beneath one of the papillary muscles. $^{2}$

Godley and colleagues specifically detected incomplete mitral leaflet closure in the vast majority of patients with mitral valve regurgitation after myocardial infarction. ${ }^{2}$ However, these investigators studied a specific group of patients with clinically important papillary muscle dysfunction. In subsequent studies using pulsed Doppler, it was shown that incomplete mitral leaflet closure is associated with raised left ventricular filling pressure and is not specific for the subset of patients with papillary muscle dysfunction or acute myocardial infarction. Kinney and Frangi, ${ }^{3}$ studying 73 patients with incomplete mitral leaflet closure, found that only $10 \%$ of them had acute myocardial infarction. The most important determining factor for the occurrence of incomplete mitral leaflet closure was the presence of mitral valve "B bumps" on $\mathrm{M}$ mode, which is typically associated with raised left ventricular end diastolic pressure and an increase in left ventricular end diastolic dimension in both dilated and ischaemic cardiomyopathy. ${ }^{3}$ Kaul and colleagues conclusively showed that incomplete mitral leaflet closure was related to reduced left ventricular function. ${ }^{7}$ Thus the distance between the mitral leaflet coaptation point and the mitral annular plane was correlated with left ventricular and mitral annulus size, but fractional shortening of the left ventricle was the only predictor of the presence of incomplete mitral leaflet closure on multivariate analysis. ${ }^{7}$ Significantly, Kaul and colleagues did not find any patients with incomplete mitral leaflet closure and poor left ventricular function or regional dyskinesis. ${ }^{7}$ Our findings should be viewed from a similar perspective.

Many investigators dealing with this subject have been particularly concerned with mitral regurgitation, often including patients or experimental animals with this abnormality as their starting point. Thus Otsuji and colleagues recently studied dogs with experimental segmental ventricular dysfunction and found that the left ventricular sphericity index but not the ejection fraction was a predictor of the severity of mitral regurgitation. ${ }^{13}$ These investigators also found that incomplete mitral leaflet closure area — which corresponds to our CPMAincreased with chronic regurgitation concomitantly with an increase in left ventricular dimension and a decrease in ejection fraction; however, these changes were not significantly correlated. In agreement with our results, Otsuji and colleagues found that segmental ischaemic left ventricular dysfunction was not associated with an important degree of mitral regurgitation. Sabbah and colleagues similarly found no relation between regional fractional area of shortening of left ventricular wall segments and functional mitral regurgitation. ${ }^{14}$

Ennezat and colleagues recently reviewed the subject of functional mitral regurgitation. ${ }^{15}$ According to these investigators, and to Goldstein and co-workers, ${ }^{16}{ }^{17}$ the pathophysiology of valvar regurgitation is determined by the degree of spherical dilatation of the left ventricle which results in apical displacement of the valve coaptation point. Papillary muscle dysfunction is a secondary phenomenon. Our results concur with this. Indeed, we found that patients with coronary artery disease and localised akinetic areas had the same CPMA value as patients with global left ventricular dysfunction (table 1).

The common characteristic of all our patients, regardless of the aetiology of their heart failure, was compromised contractility and ventricular dilatation. With respect to left ventricular and mitral annular dimensions our results are in substantial agreement with those of Kaul and colleagues. ${ }^{\text {? }}$

In patients with left ventricular dysfunction, CPMA as a measure of incomplete mitral leaflet closure was related to both the size and the function of the left ventricle. This finding supports the hypothesis that incomplete mitral leaflet closure is a result of dilatation and poor function, and not of outward pulling of the papillary muscle in systole.

In our study, CPMA was well correlated with accepted indices of systolic function (ejection fraction, fractional shortening) in both dilated cardiomyopathy and coronary artery disease. However, some important differences should be noted. In the group with dilated cardiomyopathy the correlations were on the whole much stronger than in the group with coronary artery disease, especially with respect to mitral annulus diameter. This difference stems from the segmental ventricular abnormalities in coronary artery disease and supports the global dysfunction theory.

After administration of an inotrope (dobutamine), contractile function improved and the distance between the mitral annulus plane and the coaptation point of the mitral leaflets (that is, the CPMA) decreased, further supporting our hypothesis that this is an index of systolic function.

Another significant observation was that the CPMA difference before and after dobutamine was not correlated with differences in left ventricular and mitral annulus dimensions before and after dobutamine, but only with differences in contractility. Thus contractility changes have a stronger influence on CPMA than changes in geometry. The fact that differences in fractional shortening and ejection fraction before and after preloading (by leg lifting) were not related to differences in CPMA before and after preloading - in contrast to the findings with dobutamine-suggests that the increased contractility generated by these two manoeuvres is achieved through 
separate mechanisms. The ejection fraction was increased and the CPMA decreased by both dobutamine administration and leg lifting. However, the left ventricular end diastolic dimension was decreased by dobutamine but increased by leg lifting, though both manoeuvres decreased the CPMA.

\section{Conclusions}

We believe that the study of CPMA will increase our knowledge of the anatomical and functional aspects of left ventricular dysfunction. Further information might be obtained by using other interventions such as decreased preload or increased afterload. Correlating CPMA measurements with Doppler tissue characteristics could give additional valuable insights.

\section{Authors' affiliations}

S E Karagiannis, G T Karatasakis, N Koutsogiannis,

G D Athanasopoulos, D V Cokkinos, First Cardiology Department, Onassis Cardiac Surgery Centre, Athens, Greece

\section{REFERENCES}

1 Ogawa S, Hubbard FE, Mardelli TJ, et al. Cross-sectional echocardiographic spectrum of papillary muscle dysfunction. Am Heart J 1979:97:312-21.

2 Godley RW, Wann LS, Rogers EW, et al. Incomplete mitral leaflet closure in patients with papillary muscle dysfunction. Circulation $1981 ; 63: 565-71$.

3 Kinney EL, Frangi M. Value of two-dimensional echocardiographic detection of incomplete mitral leaflet closure. Am Heart J 1985:109:87-90.

4 Dent JM, Spotnitz WD, Nolan SP, et al. Mechanism of mitral leaflet excursion. Am J Physiol 1995;269:H2100-8.
5 Kaul S, Spotnitz WD, Glasheen WP, et al. Mechanism of ischemic mitral regurgitation. An experimental evaluation. Circulation $1991 ; 84: 2167-80$.

6 Dent JM, Spotnitz WD, Kaul S. Echocardiographic evaluation of the mechanisms of ischemic mitral regurgitation. Coron Artery Dis 1996; 7: 188-95.

7 Kaul S, Pearlman JD, Touchstone DA, et al. Prevalence and mechanisms of mitral regurgitation in the absence of intrinsic abnormalities of the mitral leaflets. Am Heart J 1989;1 18:963-72

8 Otsuji Y, Gilon D, Jiang L, et al. Restricted diastolic opening of the mitral leaflets in patients with left ventricular dysfunction: evidence for increased valve tethering. J Am Coll Cardiol 1998;32:398-404.

9 Oki T, Fukuda N, luchi A, et al. Possible mechanisms of mitral regurgitation in dilated hearts: a study using transoesophageal echocardiography. Clin Cardiol 1996;19:639-43.

10 Sciller NB, Shah PM, Crawford M, et al. Recommendations for quantitation of the left ventricle by two-dimensional echocardiography. J Am Soc Echocardiogr 1989;2:358-67.

11 Thomas L, Foster E, Hoffman Jl, et al. The mitral regurgitation index: an echocardiographic guide to severity. J Am Coll Cardiol 1999;33:2016-22.

12 Weyman A. Principles and practice of echocardiography, 2nd ed. Philadelphia: Lea and Febiger, 1994:432-41.

13 Otsuji Y, Hand Schumacher MD, Lien Cohen N, et al. Mechanism of ischemic mitral regurgitation with segmental left ventricular dysfunction: three-dimensional echocardiographic studies in models of acute and chronic progressive regurgitation. J Am Coll Cardiol 2001;37:641-8.

14 Sabbah HN, Rosman H, Kono T, et al. On the mechanism of functional mitral regurgitation. Am J Cardiol 1993;72:1074-6.

15 Ennezat P-V, Logeart D, Lachmann J, et al. [L'insuffisance mitrale fonctionnelle: une valvulopathie negligée?] Arch Mal Coeur 2001:94:1173-9.

16 Kono T, Sabbah HN, Rosman H, et al. Left ventricular shape is the primary determinant of functional mitral regurgitation in heart failure. J Am Coll Cardiol 1992:20:1594-8.

17 Nass $\mathrm{O}$, Rosman $\mathrm{H}$, al-Khaled N, et al. Relation of left ventricular chamber shape in patients with low $(<$ or $\geqslant 40 \%$ ) ejection fraction to severity of functional mitral regurgitation. Am J Cardiol 1995;76:402-4.

\section{IMAGES IN CARDIOLOGY}

\section{Spontaneous dissection of left atrium presenting as pulmonary oedema}

A 28 year old man presented to the emergency department with a history of sudden onset dyspnoea of two days' duration following a brief argument with his employer. On examination the patient was experiencing class 4 shortness of breath, with bilateral diffuse coarse rales in both lung fields. He had a grade 3/6 soft blowing early systolic murmur in the apex radiating to the axilla. The ECG was unremarkable. Chest $x$ ray showed bilateral fluffy shadows in both lung fields suggestive of pulmonary oedema. Heart size was normal. Two dimensional echocardiographic study showed that the annulus of the mitral valve was elevated by the dissection, with the entry point just below the posterior mitral leaflet and the exit point in the free wall of the left atrium into the cavity. Turbulent flow is seen entering the false

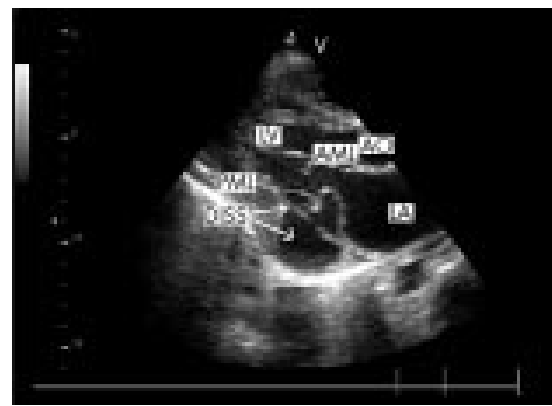

Parasternal long axis image showing a cavity in the wall of the left atrium elevating the mitral annulus. $\mathrm{AML}$, anterior mitral leaflet; AO, aorta; DISS, dissection; LA, left atrium; LV, left ventricle; PML, posterior mitral leaflet.



Transoesophageal image showing the entry point for the false cavity just below the mitral annulus at the attachment of posterior mitral leaflet. lumen and entering the left atrial cavity through the exit point. The echocardiographic grading of the mitral regurgitation thus produced was severe. The patient was treated with oxygen, diuretics, glyceryl trinitrate, and angiotensin converting enzyme (ACE) inhibitors. The patient improved within 48 hours and after seven days his shortness of breath had improved to class 2 .

The patient is to undergo cardiac surgery and is being treated with ACE inhibitors.

\section{G M Reddy J K Babu \\ S P Kumar} gokulreddy@yahoo.com

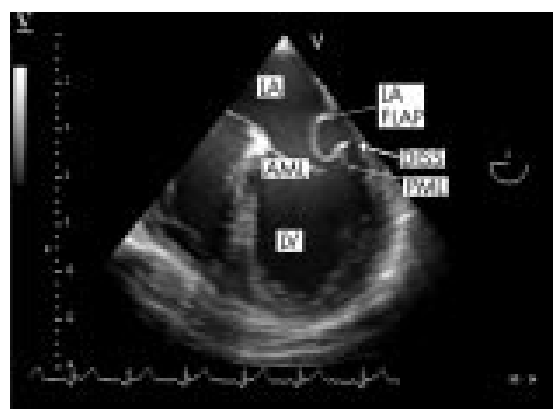

Transoesophageal image showing the entry point and the free flap of the left atrium. 